\title{
ENTRE EL SACRIFICIO Y EL DISFRUTE: PERCEPCIÓN SOBRE EL FUTURO DE JÓVENES URBANOS COSTARRICENSES
}

\author{
TATIANA BEIRUTE BREALEY*
}

\begin{abstract}
RESUMEN
Reconocer que «soñar en grande» no es una capacidad a la que todos los jóvenes puedan acceder es fundamental para lograr sociedades más inclusivas, pues permite comprender que algo tan personal como los sueños sobre el futuro, se ven limitados o potenciados por las condiciones sociales. Este trabajo analiza las aspiraciones y expectativas sobre el futuro de jóvenes urbanos costarricenses provenientes de distintos niveles de exclusión social, concluyendo que las capacidades de estos se ven influenciadas por las condiciones externas en sus aspectos más objetivos, pero que además hay elementos en lo intersubjetivo que reproducen condiciones desventajosas para su desarrollo humano.
\end{abstract}

PALABRAS CLAVE: JUVENTUDES, DESARROLLO HUMANO, EXCLUSIÓN SOCIAL

* Magíster en Desarrollo Humano por la Facultad Latinoamericana de Ciencias Sociales (FLACSO) en Argentina. Licenciada en Sociología por la Universidad de Costa Rica. Consultora y docente de la Universidad de Costa Rica. E-Mail: tatianabeirute@gmail.com. 


\title{
ENTRE O SACRIFÍCIO E O DESFRUTE: PERCEPÇÃO SOBRE O FUTURO DE JOVENS URBANOS COSTA-RIQUENSES
}

\begin{abstract}
RESUMO
Reconhecer que «sonhar grande» é uma capacidade que não todos os jovens podem ter é fundamental para conseguir sociedades mais inclusivas porque permite compreender que uma coisa tão pessoal como são os sonhos sobre o futuro estão limitados ou potenciados pelas condições sociais. Este trabalho analisa as aspirações e expectativas sobre o futuro de jovens urbanos costariquenses de diferentes níveis de exclusão social, concluindo que as capacidades deles estão influenciadas pelas condições externas em seus aspectos mais objetivos e que além disso há elementos no intersubjetivo que reproduzem condições desvantajosas para seu desenvolvimento humano.
\end{abstract}

\section{PALAVRAS CHAVE: JUVENTUDES, DESENVOLVIMENTO HUMANO, EXCLUSÃO SOCIAL}

\section{BETWEEN SACRIFICE AND JOY: PERCEPTIONS ON THE FUTURE OF URBAN YOUNG PEOPLE FROM COSTA RICA}

\begin{abstract}
To acknowledge that «dream big» is a feasible paradigm for some youngsters only is crucial to achieve a more inclusive society as it allows understanding that something as personal as dreams about future can be either fostered or hindered by social conditions. This work analyzes the hopes and expectations of urban young people from Costa Rica from diverse social strata, concluding that, from an objective perspective, their capacities are conditioned by external factors but there are intersubjective elements reproducing unfavorable conditions for their human development as well.
\end{abstract}

KEY WORDS: YOUTHS, HUMAN DEVELOPMENT, SOCIAL EXCLUSION 


\section{INTRODUCCIÓN}

LAS JUVENTUDES SON MÚLTIPLES y diversas. Si bien como grupo social «encarnan los cambios contemporáneos más fuertes» (Krauskopf, 2011), no puede hablarse de una única juventud pues cada una proyecta su futuro en función de la particularidad de su presente, y se plantan como actores de la globalización con un set de herramientas diferenciado.

Las condiciones objetivas en las que se desarrollan los jóvenes son diferentes $\mathrm{y}$, vinculadas a estas, también lo son sus construcciones subjetivas, las cuales son igualmente relevantes en la medida en que reflejan las dinámicas desiguales de inclusión/ exclusión social de la sociedad. El presente trabajo busca analizar estas condiciones subjetivas expresadas específicamente en las aspiraciones y expectativas sobre el futuro de distintos jóvenes y su vínculo con las condiciones objetivas que estos experimentan. Forma parte de una investigación más amplia denominada «¿Con qué soñás? Expectativas y aspiraciones sobre el futuro de jóvenes urbanos costarricenses» (Beirute, 2012), cuyo objetivo principal fue analizar las aspiraciones y expectativas sobre el futuro de jóvenes urbanos costarricenses, en función del nivel de inclusión o exclusión en el que se desarrollaban. Las dimensiones sobre el futuro que se analizaron fueron el nivel educativo, el tipo de empleo, el nivel socioeconómico, el lugar donde vivían y vivirán, el consumo cultural, la percepción general que tenían sobre su futuro y la trayectoria imaginada. En este artículo se hará referencia a los resultados encontrados en los últimos dos aspectos mencionados.

El saber si «soñar en grande» es un derecho al que pueden acceder solo unos, y si el abanico de capacidades con el que cuentan los jóvenes difiere según las condiciones sociales, es fundamental para lograr sociedades más inclusivas pues significa no solo comprender que las dinámicas sociales desiguales influyen en las subjetividades, limitando desde muy temprano el desarrollo humano, sino que además sirve de instrumento para proponer medidas más multidimensionales al tema de la desigualdad y la exclusión social llamando la atención sobre focos y fenómenos de la realidad poco estudiados

\section{DESDE DÓNDE SE PLANTEÓ EL ANÁLISIS}

La investigación que da pie a este artículo se fundamentó en tres perspectivas teóricas principalmente. El paradigma del desarrollo humano 
y el enfoque de las capacidades establecen que una de las partes fundamentales del bienestar humano es que las personas tienen el derecho de tener la libertad de contar con diferentes opciones para lograr ser o hacer lo que deseen hacer, pues al final de cuentas el fin principal del desarrollo es la «... expansión de las libertades reales de que disfrutan los individuos» (Sen, 2000:55).

Dentro del enfoque de las capacidades hay tres conceptos centrales. Los funcionamientos son aquellas opciones, funciones y realizaciones que las personas hacen o lo que son. (Sen, 2000). Las capacidades son $« \ldots$ las diversas combinaciones de funciones que pueden conseguir las personas... es un tipo de libertad: la libertad fundamental para conseguir distintas combinaciones de funciones (o, en términos menos formales, la libertad para lograr diferentes estilos de vida)» (Sen, 2000:99-100). Como el mismo Sen señala, los funcionamientos refieren a los logros reales que obtienen las personas a lo largo de su vida, mientras que las capacidades tienen relación con la libertad para lograrlos. (Sen, 2000a). Por último, la agencia refiere a la capacidad de las personas de actuar e influir sobre su desarrollo y bienestar.

Particularmente la noción de capacidades es la que explica la importancia de analizar y prestar atención a las aspiraciones y expectativas sobre el futuro de los jóvenes, pues estas son finalmente una forma de capacidades; es decir, son una forma de libertad. Para comprender mejor esta relación es importante comprender que las capacidades no son lo mismo que las oportunidades u opciones. El término capacidades, o el también utilizado «libertades reales», es más complejo, pues no incluye cualquier tipo de oportunidades u opciones, sino aquellas que los individuos valoran y tienen razones para valorar. Esto significa que no es tan importante tener un grupo numeroso de oportunidades, si no son valiosas; es mejor tener un número reducido de oportunidades valiosas; es decir, de capacidades. Asimismo, el pensar en opciones u oportunidades por sí mismas, hace pensar en aspectos puramente individuales, cuando por el contrario las capacidades tienen una estrecha relación con el contexto en el que se encuentran (Ugaz, 2010).

Conocer las aspiraciones y expectativas sobre el futuro de los jóvenes, resulta importante para conocer su libertad real - libertad entendida como se define en este enfoque-. Esto porque su configuración no solo remite a sus características psicológicas individuales, sino que se vincula con aspectos sociales diferenciados que limitan o potencian su efectivo desarrollo. 
Para el enfoque de las capacidades y el desarrollo humano los logros presentes (funcionamientos) de las personas no son suficientes (Sen, 2010) pues estos no abarcan todas sus libertades presentes ni futuras. El abanico de capacidades con el que cuentan las personas determina en mucho su trayectoria, y partiendo de la idea de que parte de este abanico puede conocerse al analizar las aspiraciones y expectativas sobre el futuro, es que se considera importante este estudio como un paso para potenciar el desarrollo desde una óptica diferente.

Ahora bien, las posibilidades objetivas y subjetivas con las que cuenta una persona están influenciadas por su contexto. De acuerdo al estructuralismo constructivista de Pierre Bourdieu, el vínculo entre las condiciones objetivas y las subjetividades pasa por la construcción de habitus, que ponen límites a la amplitud de las experiencias de las personas en función de las condiciones materiales y estructurales. De modo que las aspiraciones y expectativas sobre el futuro no son construcciones que respondan solo a experiencias individuales y psicológicas. Por el contrario, se construyen en diálogo con las prácticas y estructuras sociales que conforman el entorno de las personas por medio de los habitus.

Asimismo, como lo explica Bourdieu (1991), el habitus no solo permite que dentro de un determinado campo se sancionen positivamente una serie de posibilidades, sino que además se sanciona negativamente en la medida en que las posibilidades no correspondan a las condiciones objetivas, de ahí que señale que un ejemplo de este tipo de sanción es la frase «esto no es para nosotros» (1991). Esto en el caso de esta investigación ayuda a comprender el por qué algunos grupos de jóvenes ni siquiera consideren entre sus aspiraciones sobre el futuro opciones que otros ven como prioritarias. Es decir, esta perspectiva teórica implica que hasta los elementos subjetivos deben estudiarse partiendo del hecho de que las estructuras sociales tienen un papel importante sobre estos. En otras palabras las aspiraciones y expectativas sobre el futuro, de los jóvenes están marcadas por estos habitus.

La existencia de diversos habitus significa que la relación de las personas con las estructuras sociales no es la misma en todos los casos. Uno de los fenómenos que afecta esta relación son las dinámicas de inclusión y exclusión social; tercera base teórica de este trabajo. La exclusión es una forma de privación de capacidades (Calderón, 2010). Es un fenómeno multidimensional que remite a relaciones complejas. (Sojo, 2006:12) y que evidencia que en un contexto donde hay un discurso de universalidad de derechos, existen accesos y espacios 
diferenciados para ejercer plenamente la ciudadanía social, lo cual termina en el desarrollo de capacidades desiguales y/o de privación de estas. Estas experiencias diversas inciden de forma importante en la construcción de habitus diferenciados.

En síntesis, el abanico de capacidades con el que cuentan los jóvenes desde el cual construyen sus aspiraciones y expectativas sobre el futuro, se ve influenciado por las condiciones de exclusión/inclusión social en el que se desarrollan, en la medida en que los llevan a experimentar vidas muy diferentes entre sí, en términos de acceso, participación y disfrute. Las características de estas experiencias son las que finalmente llevan a construir y/o reproducir habitus que inciden en las condiciones subjetivas de los jóvenes, lo cual puede tener una incidencia positiva o negativa — según sea el caso—, sobre su desarrollo humano.

Por último, cabe señalar que en términos generales los estudios que han analizado aspiraciones y expectativas sobre el futuro de los jóvenes, coinciden en que el contexto incide en su construcción. Esto significa que las condiciones objetivas tienen alguna determinación en las subjetividades de los jóvenes que los llevan a reproducir sus condiciones sociales sin siquiera saberlo. ${ }^{1}$

\section{3. ¿CÓMO ANALIZAR LAS ASPIRACIONES Y EXPECTATIVAS SOBRE EL FUTURO DE JÓVENES URBANOS COSTARRICENSES?}

La investigación realizada fue de tipo cualitativa y se trató de un estudio de seis casos ejemplificadores. Los criterios para definirlos se derivaron de las siguientes variables: ${ }^{2}$

\section{a) El espacio socioterritorial en el que se desenvolvían los jóvenes}

Para ello se utilizaron las categorías de niveles socioterritoriales basadas en la siguiente división territorial de la Gran Área Metropolitana (GAM) (FLACSO Costa Rica, 2007):

i) Alto/alto medio: distritos que muestran un acceso privilegiado a los bienes públicos.

1 Para ver más detalles sobre estudios en esta materia ver Beirute (2012).

2 Para más detalle de cada una de las variables ir a Beirute (2012). 
ii) Medio-alto: distritos con una cantidad importante de bienes públicos de todo tipo.

iii) Medio-bajo: distritos de asentamientos de los sectores populares, y con acceso a bienes públicos menores al promedio de la GAM.

iv) Bajo-medio: la periferia urbana empobrecida.

v) Bajo: zonas de producción agrícola (esta categoría no se tomó en cuenta pues el trabajo se centró en lo urbano).

\section{b) Tipo de educación que recibían los jóvenes}

Se construyeron cinco categorías que combinan los criterios de tipo de financiamiento y características específicas de los centros así como el territorio social en el que se encuentra. A partir de esto, y con la ayuda de los datos tomados de la Megabase de Produs (Produs, 2011), las categorías planteadas fueron:

i) Colegio con condiciones óptimas: de financiamiento privado, con rendimiento académico mayor al promedio nacional y con modalidad de bachillerato internacional. Es el único colegio para el que el territorio social no fue determinante, pues lo define más el estatus que tiene en sí mismo.

ii) Colegio con buenas condiciones: ubicado en territorio social medio-alto, privado-subvencionado y con indicadores de rendimiento académico combinados.

iii) Colegio con condiciones medias: ubicado en el territorio social medio-bajo; privado-subvencionado y con indicadores de rendimiento combinados.

iv) Colegio con condiciones deficientes: se ubica en los territorios medio-bajo, es público, con indicadores de rendimiento combinados y con faltantes de infraestructura.

v) Colegio con condiciones muy deficientes: ubicado en territorio bajo-medio, es público, tiene serias deficiencias en su rendimiento académico y faltantes importantes en infraestructura.

Por último, se estableció una categoría que cumplía con el requisito socioterritorial, en este caso, que provengan de espacios bajo-medios, y que respecto a la categoría educativa esta se reflejara en su no asistencia a la educación, a lo que se sumaría el hecho de que tampoco se encontraban trabajando.

Con base en cada uno de estos criterios fueron seleccionados y ubicados seis casos ejemplificadores en las categorías que se detallan a continuación. 
- Inclusión muy favorable: jóvenes que habitaban en zonas altas/ altas-medias y asistían a un colegio con condiciones óptimas (según los criterios que se explicaron anteriormente) ${ }^{3}$ Se trataba de un colegio con Bachillerato Internacional, completamente bilingüe, de tipo privado (para ingresar las familias deben pagar una admisión que ascendía a los US \$1000).

- Inclusión favorable: jóvenes que habitaban en zonas medio-altas y asistían a un centro educativo con buenas condiciones y de financiamiento privado-subvencionado. Era aproximadamente un $60 \%$ bilingüe.

- Vulnerables a la inclusión desfavorable: jóvenes que vivían en zonas medio-bajas y asistían a un colegio en condición media, privado-subvencionado y que no era bilingüe.

- Inclusión desfavorable: jóvenes que habitaban en zonas mediobajas y asistían a un colegio en condiciones deficientes, de financiamiento público y con faltantes de infraestructura.

- Exclusión parcial: jóvenes que residían en zonas bajas-medias y asistían a un centro educativo con condiciones muy deficientes, de educación pública y con faltantes importantes de infraestructura.

- Exclusión fuerte: quienes conformaron este grupo eran jóvenes que vivían en un territorio social bajo-medio, que además se trataba de un distrito considerado de alto riesgo social (MIDEPLAN, 2010), además no asistían al colegio ni estaban insertos en el mercado laboral. Su ubicación se hizo mediante un informante clave de la zona.

A cada caso de estudio se le realizó un grupo focal. Se estableció que cada grupo debía estar conformado por estudiantes de noveno año (representaban el umbral de la retención estudiantil en Costa Rica (PEN, 2010), todos asistentes al mismo colegio según su categoría y menores de 18 años. En el caso del grupo que experimentaba un nivel de exclusión fuerte, se utilizó como criterio la edad, pues estos jóvenes no asistían al colegio. Los jóvenes pertenecientes a este grupo debían estar entre los 14 años —edad mínima requerida para estar en el noveno año- y los 17 años. Además, los otros criterios que primaron fue que vivieran en una zona baja-media y que se encontraran sin estar trabajando ni estudiando. Cabe señalar que el criterio de equidad de género no fue determinante para los objetivos propuestos en la

3 Los nombres de los centros educativos no serán divulgados pues, como parte de las condiciones para realizar el grupo focal con cada institución se acordó no divulgar el nombre de la misma, como tampoco el de los estudiantes (los nombres aquí presentados son ficticios). 
investigación que no contemplaban el análisis en función del género (aunque se buscó que se cumpliera, en la medida de lo posible).

Por último, es importante tener en cuenta que cuando se piensa en el futuro, pueden observarse al menos dos elementos que podrían ser diferentes. No es lo mismo que una persona imagine el tipo de vida que tendrá en, por ejemplo diez años (lo cual en este trabajo se llamó expectativas sobre el futuro); a que imagine el tipo de vida que le gustaría tener en diez años (lo que se entendió como aspiraciones sobre el futuro). Ambas dimensiones fueron centrales en este estudio, así como también lo fue la brecha que podía existir entre una y otra.

A continuación se presentan los principales resultados sobre el análisis de las aspiraciones y las expectativas respecto a la percepción del futuro y su trayectoria de los distintos grupos de jóvenes analizados. La información se desprende de las conversaciones que surgieron en los diferentes grupos focales realizados cuya dinámica iniciaba con la pregunta «¿cómo les gustaría estar en diez o quince años?» (aspiraciones); y luego respondiendo a la interrogante ¿cómo creen realmente que van a estar en diez o quince años? (expectativas).

En aras de una lectura más fluida, los resultados se presentan partiendo del grupo de jóvenes que vivía en condiciones de mayor exclusión, hasta los que viven en situación de inclusión más favorable. El caso de los jóvenes en condiciones de exclusión social fuerte se dejó de último pues sus particularidades los diferencian considerablemente de los demás grupos.

Por último, con el objetivo de que el/ la lector pueda comprender más el contexto desde el que se desprende este análisis es importante mencionar algunos aspectos de la realidad de la juventud costarricense de ese momento. Para el 2010, el coeficiente de Gini en Costa Rica era de 0,508 (PEN, 2011). Los problemas de la desigualdad en el ingreso afectaban a la población joven del país, donde en el $20 \%$ más pobre de los hogares (en donde vivía el $24 \%$ de la población) había más del doble de niños y adolescentes que en el $20 \%$ más rico. (Sojo, 2010). También experimentaban la exclusión en el ámbito educativo.

En el 2009, mientras que el 96,8\% de los jóvenes entre los 13 y 17 años del quintil más rico asistían a la educación tradicional, solo el $76,6 \%$ de aquellos del quintil más pobre lo hacían (PEN, 2011a). Además en los años 2003, 2005 y 2006 el 78\% de quienes accedían a la educación superior provenían de los dos quintiles de ingreso superior (PEN, 2008). Por otro lado, el desempleo juvenil urbano en el 2010 fue de $17,1 \%$ (el desempleo urbano general fue solo de 7,1\%), asimismo, el 
$12,3 \%$ de la población entre 12 y 24 años del país no estudiaba ni trabajaba.

\section{LA EXCLUSIÓN PARCIAL Y EL SACRIFICIO: VA A COSTAR PERO SERÁ POSITIVO}

El grupo de jóvenes en exclusión parcial se trató de muchachos que experimentaban el mayor grado de exclusión social entre aquellos que aún continuaban afiliados a una de las instituciones tradicionales de socialización e integración: el colegio. Eran jóvenes que residían en territorios sociales bajos-medios y que asistían a un centro educativo de educación pública con condiciones muy deficientes pues su porcentaje de repitencia y su promoción en las pruebas de bachillerato era de $25,13 \%$ y $23,25 \%$, respectivamente, mayor al promedio nacional, (PRODUS); además presentaba faltantes importantes en infraestructura.

La percepción sobre el futuro de estos jóvenes fue una combinación entre ideas de sacrificio, esfuerzo, incertidumbre y esperanza. Al consultárseles si en sus aspiraciones sobre el futuro pensaban que serían felices o si creían que iban a estar satisfechos las respuestas positivas fueron un tanto escépticas. Sin embargo el escepticismo sobre su felicidad fue aún mayor en el campo de las expectativas; el silencio predominó en estos temas. Muchos de ellos no respondieron a las preguntas sobre si serían felices y si les faltaría algo.

En general, el abanico de capacidades de estos jóvenes fue muy reducido, sobre todo respecto a la vida como esa experiencia integral que no solo incluye el estudio, el trabajo y el sacrificio. Prueba de ello fue la brecha que mostraron entre sus aspiraciones y expectativas sobre el trayecto que imaginaban para lograr lo que deseaban. A pesar de que cuando se hablaba de las aspiraciones algunos de estos jóvenes no se veían trabajando y estudiando al mismo tiempo, las dificultades de su realidad los hicieron cambiar de opinión e hicieron que en sus expectativas no hubiera duda de que tendrán que trabajar para pagar sus estudios universitarios. Pero la brecha se evidenció aún más al hablar sobre qué tipo de trabajo se imaginaban, los cuáles dejaban de ser en la rama de su profesión y se trataban de oficios con mucha menor remuneración que los mencionadas anteriormente.

Las aspiraciones sobre el futuro de estos jóvenes se centraron en aquellas dimensiones relacionadas únicamente con el esfuerzo y el sacrificio. Esto no quiere decir que no pensaran que iban a disfrutar nunca; sin embargo, al pensar en el tipo de vida que valoraban vivir, el 
disfrute y el descanso pareciera que se presentaban como un «lujo» en el cual no podían detenerse a pensar cuando la realidad se les venía encima cargada de muchas obligaciones y esfuerzos. El disfrute solo les llegará al final de todo el esfuerzo, como expresó Jorge respecto a su trayecto de vida, «Estudiar para trabajar y después pensionarse y vagabundear». ${ }^{4}$

Sin embargo, a pesar de los esfuerzos, manejaban una visión esperanzadora del futuro e imaginaban un futuro muy diferente al que vivían en ese momento sus padres, es decir proyectaron una movilidad social ascendente. De hecho, para la mayoría de ellos el solo hecho de estar cursando noveno año de secundaria ya los colocaba en una posición diferente a la de sus progenitores. Es muy positivo que estos jóvenes manejaran aspiraciones y expectativas sobre el futuro esperanzadoras, sin embargo, aquí se presenta el gran reto de qué pasa cuando lleguen a chocar con una realidad que los continúa excluyendo - falta de espacios para estudiar en las universidades públicas, falta de empleos competitivos, entre otros- Se genera frustración, se genera desapego, se genera desconfianza.

Asimismo, no se mencionaron limitaciones concretas, lo cual es muy sugerente. Claramente se trata de jóvenes cuyas condiciones objetivas les auguran un futuro difícil. Ahora bien, es posible que de la misma forma en que sus sueños no mostraron una estructura congruente porque no tenían muy claro cómo lograr lo que aspiraban, las limitaciones tampoco fueron muy evidentes probablemente porque en realidad no tenían la menor idea de cuáles serían las dificultades que tendrán que afrontar pues sus sueños son muy lejanos a su vida actual.

En síntesis, las capacidades de estos jóvenes se vieron limitadas en torno a unas pocas dimensiones de la experiencia humana; no hay tiempo para nada más. Si las libertades reales son aquellas oportunidades que las personas «tienen razones para valorar» (Sen, 2000), sería irreal pensar que estos jóvenes no valoran el disfrute de la vida y el descanso, sino que, dado que las libertades también tienen una estrecha vinculación con el contexto en el que se desenvuelven, pareciera que estos jóvenes, si quieren mejorar su situación, como así lo señalaban, de cierta forma no tienen el derecho de valorar este tipo de oportunidades, o al menos de priorizarlas. El disfrute en su futuro tiene el último puesto. Esto se debe también al peso del habitus que cae sobre

4 Las transcripciones que se presentan son literales para no alterar el sentido de lo dicho. 
ellos, pues «...las prácticas más improbables se encuentran excluidas sin examen alguno, a título de lo impensable» (Bourdieu, 1991:94).

\section{LA INCLUSIÓN DESFAVORABLE Y EL PRINCIPIO DE REALIDAD: EL SUEÑO ES SOLO UN SUEÑO}

Los muchachos que formaron parte de este grupo se caracterizaban por contar con mejores niveles de inclusión social que los jóvenes en exclusión parcial, aunque su situación precaria hacía que no pudieran considerarse más incluidos que excluidos.

En sus respuestas respecto a sus aspiraciones y expectativas sobre el futuro estos jóvenes mostraron las contradicciones que se experimentan cuando se vive el dualismo entre la exclusión y la inclusión social. Reconocieron su determinación personal, sus logros educativos, que son mayores a los alcanzados por sus padres, y las ventajas que podrían tener, como por ejemplo la ayuda familiar; pero aun así no fueron del todo optimistas sobre su futuro. Pareciera que el entorno les pesó más; las limitaciones que observaban día a día hicieron que no se permitieran esperar fácilmente un futuro positivo.

En el campo de las aspiraciones, la felicidad que percibían sobre su futuro fue completa y la percepción de satisfacción también. Sin embargo, lamentablemente este positivismo no se reflejó en sus expectativas, observándose una mezcla entre incertidumbre y el reconocimiento de un camino difícil. Ninguno se atrevió a responder afirmativamente a la pregunta de si serían felices; las tímidas respuestas solo alcanzaron unos «espero que sí», «más o menos», o un silencio. Tampoco se mostraron muy certeros en señalar si estarían satisfechos, y ante la pregunta de si les iba a faltar algo la respuesta remitió al sacrificio. Como dijo Valeria, «Si uno estudia y tiene plata no le va a faltar nada».

Al respecto es posible pensar que si en el caso de los jóvenes en condiciones de exclusión parcial la frustración y la imagen de un futuro sumamente adverso no era evidente, pues su realidad estaba muy lejana siquiera para empezar a pensar en las limitaciones, en el caso de los jóvenes en inclusión desfavorable, que al estar un tanto más incluidos tenían una idea de los obstáculos a los que podían enfrentarse, sabían muy bien que con solo su esfuerzo podía no bastar, y por eso no estaban tan seguros de que podrían alcanzar sus sueños o que podrían salir adelante.

Pareciera que en este grupo social las limitaciones del habitus se manifestaron de forma más explícita, dibujándoles un futuro más con- 
tradictorio con una diversidad de dimensiones deseadas para su vida, pero un abanico de capacidades reales muy limitado.

Tanto así fue la conciencia y el reconocimiento de la adversidad, que para estos jóvenes, al hablar de cuáles eran las diferencias entre lo que se les planteó como aspiraciones y expectativas, la respuesta fue: «todo». Como dijo Raquel, la diferencia estaba en que «Tal vez porque lo que hablamos al principio (aspiraciones) es un sueño y ahorita de lo que hablamos (expectativas) es una realidad». La realidad les da una bofeteada y los hace pensar que el sueño solo será un sueño. Lo que cambia, según Raquel, eran «Las posibilidades de que podemos llegar a tener a ese sueño» a lo que agregó Valeria, «Sí ya no tendríamos el carro, la casa, ni nada de eso». Es interesante notar que como grupo eran más negativos que individualmente. Todos estaban muy conscientes de su exclusión, pero tenían un plan que podría pintar más positivo el futuro que se imaginaban.

En términos de movilidad social, los relatos expresados por estos jóvenes mostraron continuamente la combinación entre aspectos positivos y posibles limitaciones. Por un lado, evidenciaron su orgullo por los logros alcanzados hasta ahora; sin embargo, pareciera que sus logros podrían no ser suficientes, pues a pesar de este auto reconocimiento que hacían no vislumbraron cambios mayores respecto a la realidad actual de sus padres. De hecho, ante la idea de un cambio socioeconómico en el futuro, la respuesta fue negativa, a lo que Alan añadió, «diez años es muy poco tiempo en realidad».

La frustración de la que son víctimas desde tan temprana edad, ya demostraba la limitación de las libertades reales de estos jóvenes, pero también las limitaciones que tendrán en términos de agencia, pues hay que recordar que la agencia es plural y si ellos no ven potencialidades en su grupo social y sufren de baja autoestima social, las posibilidades que tendrán de reivindicar mejores condiciones son más limitadas.

Por último cabe destacar que al igual que en el caso de los jóvenes en exclusión parcial, aunque en menor grado, los temas más relacionados con el disfrute no estuvieron tan presentes, primando en sus relatos aquellos aspectos relacionados con el trabajo y el estudio, pues su meta era salir de donde estaban ahora, y para ello lo más necesario era el esfuerzo. 


\section{VULNERABILIDAD E INCERTIDUMBRE: DEPENDE...}

Este grupo representó a los jóvenes que estaban en condiciones de vulnerabilidad de caer en inclusión desfavorable. Se trataba de aquellos que disfrutaban de ciertos grados de inclusión social pero, al mismo tiempo, las condiciones en las que se desenvolvían no eran lo suficientemente estables para poder afirmar que disfrutaban de todas las ventajas sociales que implicaba estar dentro de los incluidos en la sociedad. Formaban parte del grupo de población que pasa fácilmente de la inclusión favorable a la desfavorable.

En el campo de las aspiraciones, para ellos el futuro parecía muy positivo; sentían que serían felices, que estarían satisfechos y que no les haría falta nada. Sin embargo, al pasar al campo de las expectativas, el futuro pareció ser muy incierto. El entusiasmo con que respondieron que serían felices en su sueño, se perdió. Los que respondieron afirmativamente a la pregunta de si serían felices lo hicieron con duda, y hubo algunos que solo respondieron «depende».

La vulnerabilidad de la realidad de estos muchachos se hizo evidente desde que se inició la conversación respecto a las expectativas. Por ejemplo, Raúl en su sueño se veía siendo millonario y viviendo solo, pero a la hora de hablar de las expectativas, expresó que ya no se imaginaba solo y ante la interrogante de ¿por qué?, su respuesta fue, «Diay porque ocupamos a alguien que me ayude con la plata». La incertidumbre fue la regla en las expectativas sobre el futuro de estos jóvenes. Ninguno se atrevió a decir que estaba seguro que sería feliz y satisfecho, como sí lo hicieron convencidos cuando se trataba de las aspiraciones.

Resulta interesante, que en el caso de estos jóvenes en condiciones de vulnerabilidad, los miedos al futuro se diferenciaron de los de la mayoría de los jóvenes de los otros grupos sociales, pues se basaron principalmente en las posibles eventualidades que presenta un mundo lleno de incertidumbres y que podían llegar a afectarles: el embarazo adolescente, la inseguridad ciudadana (tener un accidente o ser víctima de un robo que los dejara con algún tipo de discapacidad), etcétera. Es decir, el miedo, como dijo Lucía, «a que las cosas no salgan como uno las planee», o como lo expresó Leonardo, «Es que uno no sabe lo que le va a pasar el día de mañana. Como dijo Raúl ahora en cualquier momento viene alguien y comienza a tirar balazos, le pega uno a uno 
en la jupa ${ }^{5}$ y ahí cae». Es en este punto donde el habitus de su condición social se hizo más evidente; la vulnerabilidad de su condición los hacía pensarse indefensos ante lo que la vida les pudiera poner en el camino.

Por otro lado, en términos de una movilidad social futura, para estos muchachos el ascenso en términos económicos no fue tan central como sí lo era el tipo de labores en las que planeaban desempeñarse, relacionado principalmente con la idea de sacrificio. De ahí que aunque no les gustara o no les llamara tanto la atención, igual continuaban estudiando. Para ellos las posibilidades que les daba el tener una vida más confortable que la de sus padres, les permitiría tener menos sacrificios y era eso principalmente en lo que querían ascender.

Fue interesante notar que en la mayoría de las veces en que se hablaba del esfuerzo que tenían que hacer para lograr lo que soñaban, algunos utilizaron expresiones que evidenciaban que ellos sabían que se trataría de un esfuerzo grande y no muy sencillo. Por ejemplo, al hablar de cómo podían llegar a cumplir sus sueños, Luis expresó, «estudiando como esclavo».

Por otro lado, al igual que los casos de los jóvenes en condiciones menos favorables, para estos muchachos la trayectoria de su futuro estaba marcada por la combinación entre estudio y trabajo, y el «lograr lo que querían» era un proceso largo. Aquí comenzaron a verse otras dimensiones en sus abanicos de capacidades más relacionadas con el disfrute y con un estilo de vida «bien». El trabajo y el esfuerzo dejaron de ser el centro y eran apenas vistos como un medio; no formaron parte de la centralidad del tipo de vida que valoraban, aunque como se mencionó, en sus relatos dejaron salir frases que mostraban que, aunque fuera inconscientemente, sabían que el esfuerzo debía ser grande. Aquí el habitus se manifestó imponiéndoles límites a sus estructuras cognitivas.

\section{LA INCLUSIÓN FAVORABLE Y LA REALIZACIÓN PERSONAL: VOY A CUMPLIR MI SUEÑO}

Quienes conformaron este grupo residían en territorios sociales medio-altos y asistían a un centro educativo privado subvencionado con buenas condiciones.

5 Cabeza. 
En este grupo sucedió una situación muy particular pues la diferencia entre la idea de aspiraciones y expectativas les causó confusión. Al igual que en los demás grupos focales, se inició la conversación hablando sobre las aspiraciones respecto al futuro. Sin embargo, cuando se pasó a hablar sobre las expectativas, los jóvenes se notaron confundidos, pues todo lo que habían señalado respecto a sus sueños se trataba en realidad de lo que efectivamente creían que iban a lograr; es decir, el factor obstáculo estaba de cierta forma tan lejano a su realidad, que la diferencia entre aspiraciones y expectativas les causó confusión. Una vez explicada la diferencia se procedió a hablar de lo que ahora sí aspiraban; sin embargo fue evidente que se trataba de temas y elementos con los que estos jóvenes podían soñar, pero que claramente no condicionarían su felicidad, pues en realidad esperaban tener todo lo que quisieran. El resto sería un «extra» que no consideraban demasiado necesario.

Respecto a la percepción del futuro en el campo de las expectativas, para ninguno de estos jóvenes existió duda alguna de que les esperaba un futuro feliz y satisfactorio La posibilidad de cumplir el futuro que se imaginaban fue casi total; se les pidió que señalaran un porcentaje de posibilidad y las respuestas rondaron entre el $80 \%$ y el $90 \%$. No había imposibles y no visualizaban obstáculos, de ahí que les costara diferenciar entre expectativas y aspiraciones.

A estos jóvenes el futuro no se les presentaba como algo a lo que hubiera que temerle, más allá del cambio que significaba tener más responsabilidades, en el sentido de que tendrían más independencia de sus padres. En términos de calidad de vida consideraban que no había mucha diferencia pues estaban completos ahora y seguirían completos en el futuro. Como explicó Lorena, «...igual ahorita no me hace falta nada. Gracias a mis papás lo tengo todo y en mi sueño me veo igual que no me va a hacer falta nada».

Por otro lado, respecto a los obstáculos para estos jóvenes nada era imposible, de ahí que no visualizaran con claridad las limitaciones. Como dijo Megan «Diay es que la verdad si uno se lo propone todo lo puede hacer. Uno mismo es el que dice no puedo hacer esto o lo otro». Esto explica el hecho de que para ellos su futuro dependía de ellos mismos y de nadie más.

Por otro lado, en consonancia con su visión positiva del futuro y su conciencia de situación privilegiada, estos jóvenes imaginaron trayectorias de vida que tomaban en cuenta la idea de irse realizando como personas en diversas dimensiones, reflejando un abanico de 
capacidades amplio. Al igual que los jóvenes de los otros colegios, la trayectoria incluía terminar la secundaria y pasar a la universidad. La diferencia aquí es que el tema laboral no estaba en el medio de estos dos pasos. Para estos jóvenes la opción de ser estudiantes de tiempo completo sí existía y la deseaban tomar. Asimismo la mayoría de sus relatos mostraban una cierta moratoria post universidad que consistía en la idea de darse un tiempo para la expansión de sus vidas después de haber completado las bases necesarias para participar de forma privilegiada de la globalización. Por ejemplo Mauricio quería viajar y Manuel decía que «Diay, bueno yo termino el colegio, quedarme un año estudiando idiomas y entrenando». Las diferencias con los jóvenes en condiciones sociales menos favorables fueron obvias.

Además se observaba una clara estructuración de sus sueños. Sabían muy bien cómo debían lograrlos, no eran metas en el aire. La trayectoria descrita por Carlos es ejemplo de ello: «Diay yo saldría del colegio, de aquí, me iría a la UCR o si no a la UACA. ${ }^{6}$ Es que de ahí salió mi prima y ella dice que es muy buena. Diay me graduaría. Luego, bueno uno se gradúa de la Universidad, y tiene ocho meses, esos ocho meses son de vacaciones y después de esos ocho meses te metes al hospital ya como interno. Entonces ya me metería como interno, sacaría la residencia, y luego me daría un tiempo de vacaciones para descansar. Y luego ya me metería a trabajar. Me gustaría trabajar ya como unos diez años y tener la plata suficiente como para abrirme la clínica, o sea como una clínica y otra clínica como de ayuda para la gente que lo necesite».

En síntesis, en términos de expectativas para estos jóvenes su futuro debía ser visto como algo integral. No se limitaba a un buen trabajo, una buena educación o una buena casa. Se trataba de todo eso y más. La vida que valoraban vivir no era solo una vida de sacrificios y esfuerzos, sino que se trataba de un disfrute de los ámbitos que la conformaban. Para ellos lo que se les venía era visto como positivo, aquí el habitus funcionaba de manera inversa a los jóvenes en condiciones desfavorables, pues lo que sancionaba era la idea de las limitaciones. Se trató realmente del ejemplo de grupo de jóvenes para el cual está hecha la globalización; esos que cuentan con las herramientas adecuadas para enfrentarla perfectamente.

6 Universidad privada. 


\section{LA INCLUSIÓN MUY FAVORABLE Y LAS DESTREZAS DE LA GLOBALIZACIÓN: MEZCLA ENTRE FELICIDAD E INSEGURIDAD}

Este grupo lo conformaron los jóvenes que experimentaban mayores niveles de inclusión social. Si bien la inclusión- exclusión social no son fenómenos duales (Calderón Umaña, 2008), podría señalarse que estos jóvenes gozaban de casi todos los niveles de inclusión a los que podría aspirar un joven costarricense.

Al hablar sobre la percepción de su futuro tanto las aspiraciones como las expectativas fueron positivas; no había brecha entre una y otra. Estaban seguros que serán felices y que estarán satisfechos. Respecto al hecho de que les faltaría algo, sí lo consideraron posible, pero tenía que ver con eventualidades que se alejaban de cualquier influencia social y que se debían a circunstancias personales. Por ejemplo, Cecilia decía: «No sé, tal vez que queramos tener hijos y no podamos». Tenían una idea clara de lo que querían para su futuro: algo similar a lo que ya tenían. Además, sabían que contaban con todos los instrumentos para lograrlo.

La condición de inclusión muy favorable de la que gozaban estos jóvenes les abre la puerta a un mundo lleno de posibilidades y alternativas. Es decir, el conjunto de capacidades respecto su futuro es muy amplio. Sin embargo, formar parte de un momento histórico tan competitivo y exigente como el actual, los hacía no solo ser el grupo social más apto para enfrentar los retos de la globalización, sino también el más consciente de su exigencia. De ahí que extrañe que una de las características más particulares de este caso de estudio, y que fue un tema recurrente en todo el grupo focal, era que se trataba de un grupo que mostraba muchos miedos e inseguridades respecto al futuro, relacionados principalmente con la toma de decisiones correctas para poder llegar a ser exitosos.

Por ejemplo, Silvia explicó: «Yo todavía no sé qué quiero estudiar, entonces a veces como que me da miedo no estudiar al final lo que yo quiero, o sea que elija lo equivocado...». Pareciera que sentían una cierta presión por vivir de acuerdo a sus propias expectativas y aspiraciones. El habitus que manejaban apareció recordándoles que se espera mucho de ellos. Y además mostraba que se trataba de jóvenes que sentían la presión de un orden mundial en donde, como lo expresa Bauman (2011), la forma más eficiente de control social ha pasado a basarse en la inseguridad. 
Sin embargo, a pesar de los miedos e inseguridades que tenían respecto a tomar las decisiones correctas para un mejor futuro, aun así no vislumbraban ni la mínima posibilidad de que no fueran a ser felices y satisfechos.

Por su parte, la trayectoria de estos jóvenes se relacionó mucho con el ámbito profesional pero al mismo tiempo tenían muy presente la combinación con el disfrute. Fue claro que para ellos el periodo que vendría luego de salir del colegio no se trataba de una etapa preparatoria para una vida de disfrute si no que era parte del disfrute mismo de la vida. Algunos consideraban la idea de darse un tiempo libre; otros no deseaban hacerlo recién saliendo del colegio, sino hasta que ellos mismos pudieran costearlo.

Por último, la idea de trabajar para pagarse los estudios no estaba entre sus planes. Aunque sí consideraban trabajar mientras estudiaban, no por una necesidad, como la de los jóvenes en condiciones menos ventajosas, pero sí para costearse comodidades que les gustaría poseer. Por ejemplo, Silvia expresó que, «No para los estudios pero sí para las otras necesidades. Como que mis papás sí me van a pagar los estudios, pero me van a decir, como 'diay, no, si quiere un apartamento se lo tiene que conseguir'»; y Cecilia «O páguese su gasolina o algo».

En síntesis, el abanico de posibilidades era muy amplio. Fue evidente que se trató de jóvenes a los que el sistema social tenía totalmente incluidos. Si bien en la intersubjetividad de este grupo la idea de esfuerzo sí es considerada, la de sacrificio no estaba presente; y si lo estaba, era vista como parte de lo que debían hacer para tener determinado elemento que querían, pero siempre con el «lujo» de la elección. Dado que para ellos el dinero y lo material no era central, pues era algo con lo que contaban ahora y no ponían en duda que tendrían en el futuro, la idea de ser exitosos incluía más que el dinero. Como lo dijo Silvia, el éxito «no es solo como tener plata, también es ser feliz en lo que hago».

\section{LA EXCLUSIÓN FUERTE Y EL PRESENTISMO: VIVIR EL MOMENTO}

Quienes conformaron este grupo eran jóvenes que vivían en un territorio social bajo medio, que además se trataba de un distrito considerado de alto riesgo social (MIDEPLAN, 2010) no asistían al colegio ni estaban insertos en el mercado laboral. Su ubicación se hizo mediante un informante clave de la zona. Se optó por colocar como último caso pues la dinámica que mostraron se diferenció bastante respecto a los 
otros grupos de jóvenes de estudio, principalmente porque no existió una idea de futuro claramente presente.

Para estos jóvenes el futuro era algo en lo que pensaban a veces; no muy frecuentemente. Esto no solo fue expresado por ellos explícitamente sino que además se evidenció en la ausencia de algún tipo de plan. Cuando se les preguntó dónde pensaban que podrían estar dentro de diez o quince años, la respuesta de André fue representativa de la sensación grupal: «No sé, no sé ni donde voy a estar mañana... yo voy viendo a ver qué pasa en el día, yo no me levanto a pensar qué voy a hacer hoy, yo me levanto y veo qué pasa».

La idea de un futuro satisfactorio era borrosa. El presentismo fue el tinte de su discurso, y este presente se vinculaba con la idea del disfrute. Al abandonar los mecanismos formales de socialización e integración social estos jóvenes optaron por dejar de tener una vida sacrificada. Claramente esta no fue una decisión solamente individual: las condiciones sociales de fuerte exclusión social en las que se desenvolvían los habían llevado a tener que escoger entre una y otra vía. Para ellos el derecho a disfrutar de una buena calidad de vida, mediante los mecanismos tradicionales, no era una opción real. De momento habían asumido la idea de la juventud como ausencia de responsabilidades, y donde debía primar el disfrute; lo demás sería problema de después. Sabían que eventualmente tendrían que asumir responsabilidades para las cuales al menos deberían de trabajar (por lo menos al decidir independizarse de sus padres) pero no sabían ni cuáles, ni cuándo, ni cómo realmente las asumirían. Como dijo André, «No yo prefiero no pensar [se ríe]. Es que yo prefiero no pensarlo para no preocuparme, ahorita, si yo todavía soy joven».

Uno de los principales obstáculos que veían era su condición socioeconómica. Como dijo André, «Sin plata no llego ni a la esquina». La centralidad del dinero fue clara en su discurso, y no era de extrañar. Con su abandono del estudio y el vínculo tan pequeño que mostraron respecto a sus familias, se evidenció que ya habían dejado de confiar en las instituciones tradicionales en las que se apoyan muchos de los jóvenes en esa etapa de su existencia, para lograr una mejor calidad de vida. De modo que en este contexto el dinero sería el que vendría a representar ese nivel anhelado, y era el factor que determinaría, de cierta forma, su felicidad.

Para estos jóvenes la vida que llevaban no era considerada difícil, de hecho, a pesar de que no tenían el dinero para poder adquirir todo 
lo que quisieran (por lo que se veían obligados a «negociar» ${ }^{7}$ ), sus necesidades básicas estaban satisfechas, lo que les permitía hacer lo que más disfrutaban, que André lo definió como «La juventud. Andar con los compas». Una mirada a su rutina diaria (muy similar en todos los casos) era reflejo de esto. Por ejemplo, André explicó: «Bueno yo me levanto como 9:30 o 10:00, ya si me levanto tarde es a las 11:00. Me levanto, prendo el tele, me quedo viendo. Como a las 12:30 me levanto, me baño, me mudo, almuerzo, voy para la calle; llego como a las 4:00... Luego entro, como, vuelvo a salir y me meto». La de Keryl era: «Yo siempre me levanto tarde, como a las 11:30 o 12:00. Escucho música, pongo el play, me quedo jugando tarde; después me baño, almuerzo y salgo a la calle. También a hacer papel. ${ }^{8}$ Después nada más entro, como, y vuelvo a salir».

Respecto a su futuro, no existieron trayectorias planeadas o soñadas. Sí deseaban tener dinero suficiente para, ojalá, comprarse un carro, independizarse de sus padres y poder comprar ropa y otras comodidades materiales. La forma de lograrlo era la que no habían reflexionado. Sabían que sería bueno estudiar, pero no creían tener las cualidades para ello; sabían que debían trabajar, pero no tenían idea de dónde. Es decir, sabían cuáles serían las típicas respuestas, pero las descalificaban (al menos de momento) por sus actitudes personales. Como dijo Sergio «Tal vez conseguir un trabajillo ahí, fresco, ${ }^{9}$ quien sabe, qué pereza. Es que no estoy acostumbrado a hacer nada».

Para estos jóvenes el futuro no era un tema; la búsqueda de identidad y de sobrevivencia en un mundo lleno de objetos que les eran negados por falta de recursos, y que deseaban tener, colmaba sus preocupaciones diarias. El futuro ya vendría. Por ello al preguntárseles respecto a qué pensaban del futuro las respuestas más comunes fueron: «yo casi nunca»; «yo nada», y «yo no pienso en eso». La verdad es que las culturas juveniles excluidas «... se posicionan con respecto al mundo desde un presente en el que se renuncia a esa idea de porvenir, de futuro. La realidad se experimenta en lo cotidiano, como una

7 Así se refirieron a la forma que tenían para conseguir dinero. Lo que hacían es que buscaban personas que les dieran objetos para vender (sin indagar sobre su procedencia). Por ejemplo, un teléfono celular, y lo vendían a un precio mayor al que se los dieron y de esta manera obtenían alguna ganancia.

8 Pasar tiempo con los amigos en una esquina sin estar haciendo ninguna actividad en especial.

9 Relajado. 
realidad sin historia, sin proyecto» (Reguillo, 1994:173) Esto no es de extrañar. Estudios internacionales mostraron que las personas con aspiraciones muy altas respecto a su nivel de vida actual, tienen muy pocos incentivos para invertir en estas, pues las diferencias seguirán siendo grandes, aún después de su esfuerzo (Genicot y Raj, 2009).

\section{Conclusiones}

En términos de aspiraciones, para todos estos jóvenes existió la de tener un futuro feliz y satisfactorio. Si bien algunos más convencidos que otros, el sentimiento positivo primó, con la excepción de aquellos en exclusión social fuerte, que prefieren no pensar en su futuro.

Las diferencias comenzaron a aflorar al hablar de la percepción del futuro esperado, siendo los sectores del medio los más escépticos y de cierta forma desesperanzados; esto porque eran los que estaban más conscientes de su fragilidad social. Para los jóvenes en exclusión parcial, ese futuro esperanzador con una profesión, una buena casa, familia y dinero, era tan lejano a su realidad actual que aún no podían establecer concretamente los obstáculos a los que podrían enfrentarse, pues ese mundo de inclusión les era muy ajeno. En cambio, para los jóvenes en inclusión desfavorable y en vulnerabilidad la experiencia de vivir en espacios que los incluían, pero que al mismo tiempo los rechazaban, hacía que supieran que el camino que les esperaba no sería fácil. Sin embargo, la diferencia entre ambos es que mientras que los jóvenes en condiciones de inclusión desfavorable estaban más excluidos que incluidos, primaba la extrema conciencia de la realidad en la que se desarrollaban - más que en ningún otro grupo-; mientras que los jóvenes en vulnerabilidad, en ocasiones estaban más incluidos y en otras menos incluidos, pero nunca tan cerca de la exclusión social; por lo que en ellos lo que primó fue la incertidumbre.

Por su parte, en el caso de los grupos más incluidos, también se observó una diferencia. Mientras que los muchachos en inclusión favorable visualizaban un futuro muy prometedor, los jóvenes en condición muy favorable lo percibieron con temor. Esto porque en los primeros existía todavía la posibilidad de un ascenso, que los impulsaba a invertir en sus metas para lograrlas, mientras que en los segundos, que ya gozaban de mucha inclusión social y contaban con las herramientas por excelencia para enfrentar los desafíos de los tiempos que vivían, el mantener ese estatus, y el no equivocarse, les generaba miedo. Sin embargo esta incertidumbre era diferente a la de los jóvenes en 
condiciones de inclusión desfavorable y en vulnerabilidad, pues no se cuestionaba tanto la idea de que iban a ser felices, sino que dudaban de su capacidad de estar al corte de sus propias expectativas. Aun así, ambos grupos reflejaban claramente que vivían un proceso real de individualización, cuyas características son «que sean ellos mismos, que se realicen, que sean lúcidos y sinceros con sí mismos, que elijan lo que quieren ser, no importa equivocarse, hay que descubrir y realizar los sueños, alcanzar el máximo de placer, no está bien sufrir, no hay que dejar de velar por su seguridad, ser libre, pero sin errar pues les espera la muerte social, ser un supernumerario». (Bajoit, cit. por Krauskopf, 2010:33). Por último, en el caso de los muchachos en condiciones de exclusión social fuerte, las necesidades de inclusión son tantas en el presente, que se ven imposibilitados, y también optan por no pensar en el futuro.

Uno de los rasgos más interesantes encontrados a partir de este análisis fue la de la idea del sacrificio versus la idea del disfrute. De acuerdo a Bourdieu, el mundo práctico constituido a través del habitus es un mundo de «caminos a seguir» (Bourdieu, 1991). El análisis de los casos de estudio de esta investigación permitió observar claramente tres tipos de caminos percibidos por estos jóvenes, en función de su condición social, que marcaban su intersubjetividad como grupo e influyó en sus abanicos de capacidades: el del sacrificio y esfuerzo constante, en los grupos de exclusión parcial, de inclusión desfavorable y en menor medida en los jóvenes en vulnerabilidad; y el camino de la realización personal y el disfrute, en los jóvenes en condiciones de inclusión favorable y muy favorable. Hasta aquí se muestra que conforme hay mayor exclusión, más se visualizan los próximos años como pasos a seguir para lograr una meta futura; mientras que a mayor inclusión, más imagen del futuro como etapas de la vida valorables cada una en sí misma; es decir no se camina sacrificadamente a una meta, sino que cada paso es una meta que piensan disfrutar. Un tercer camino a seguir, que se distancia completamente de los dos anteriores, sería el de los jóvenes en exclusión social fuerte, quienes optaron por la desafiliación y el presente.

De acuerdo a Sen, una «buena vida» es en parte un tipo de vida de elección genuina, y no una en la que la persona se ve forzada a llevar un tipo de vida (Alkire, 2005:120-121). Lo que se observa al comparar a los distintos estudios de caso de este trabajo, es que hay jóvenes cuyas condiciones sociales los fuerzan a elegir estilos de vida que no necesariamente son los que más valoran, pero que constituyen 
los que creen que tienen las posibilidades de llegar a tener. Esto se evidencia, particularmente, en el caso de las brechas entre aspiraciones y expectativas sobre el futuro de los jóvenes en condiciones de exclusión parcial, inclusión desfavorable y, en algunos casos, en aquellos en condiciones de vulnerabilidad. Esta especie de coerción, producida por los habitus, que se ven reforzados por las condiciones de exclusión social, no se observó en los casos de las aspiraciones y expectativas sobre el futuro de los jóvenes en condiciones de inclusión favorable y muy favorable.

Por otro lado, como señalan Krauskopf y Reguillo, las juventudes actuales se enfrentan a la paradoja social de «la incertidumbre como la única certidumbre» (Krauskopf, 2010:36), aunque esta no se percibe de la misma manera. Florencia Saintout (2007), propone diferentes incertidumbres derivadas de las distintas posiciones sociales de las que provienen los jóvenes. Por un lado está la incertidumbre como celebración, de la que forman parte los jóvenes en condiciones de inclusión favorable y muy favorable, en la que la falta de certezas del contexto actual se experimenta como un abanico de opciones para elegir, a la que se suma una fuerte presencia de la individualidad - $\mathrm{a}$ pesar de que en ocasiones les cause algo de miedo no estar al corteUn segundo tipo es la que la autora denomina «el dejarse llevar», de la que forman parte los jóvenes en condiciones de vulnerabilidad y en la que, a pesar de las capacidades personales, se dota de un peso importante al azar. Por último, la autora propone la noción de incertidumbre como adversidad, que significaría una presencia de cierto pesimismo - en menor o mayor grado- en los jóvenes en condiciones de inclusión desfavorable y de exclusión parcial; y una pérdida del impulso y la motivación, en los jóvenes en condiciones de exclusión social fuerte, que han optado por «....revolverse en la intemperie y ver qué resulta, sin demasiadas esperanzas» (Saintout, 2007:214).

En términos generales, puede afirmarse que, al menos en los casos de estudio planteados en esta investigación, sí se observó un problema de desarrollo humano en tanto que pudo evidenciarse que las libertades y capacidades de los jóvenes, no solo se ven influenciadas por las condiciones externas en sus aspectos más objetivos y concretos, sino que además hay elementos importantes que suceden en lo intersubjetivo, que pueden reproducir condiciones desventajosas para su desarrollo humano y la potenciación de sus libertades. Esta situación es lamentable pues se trata de una forma inconsciente de mantener un status quo desigual y de una continua reproducción del acci- 
dente de nacimiento (Kliksberg, 2008) que afecta a los jóvenes no solo en sus condiciones materiales sino además en aquello que todos tienen derecho a tener sin límites: sus sueños y aspiraciones.

SAN JosÉ (COSTA RICA), JULIO 2014

RECIBIDO: JULIO 2015

ACEPTADO: SEPTIEMBRE 2015

\section{REFERENCIAS BIBLIOGRÁFICAS}

AlKIRE, SABINA (2005): «Why the Capability Approach?» Journal of Human Development, Vol. 6, Nº1.

BAUMAN, ZYGMUNT (2011): La sociedad sitiada. Buenos Aires: Fondo de Cultura Económica.

BEIRUTE, TATIANA (2012): «¿Con qué soñás? Expectativas y aspiraciones sobre el futuro de jóvenes urbanos costarricenses». Tesis de Maestría. FLACSO Argentina, Buenos Aires.

BourdiEU, PIERRE (1991): El sentido práctico. Madrid: Taurus Ediciones.

CALDERÓn, FERNANDO (2012): «Clase 7. Los jóvenes como actores del desarrollo en el MERCOSUR: Inclusión con igualdad. Reconocimiento, redistribución y participación. La cultura como plataforma de inclusión. La política como espacio público para la inclusión. Curso de Desarrollo Humano II. Maestría en Desarrollo Humano». FLACSO Argentina.

FLACSO, COSTA RICA (2007): «Informe final del estudio social para PRUGAM». San José: FLACSO.

GENICOT, GARANCE y DEBRAJ RAY (2009): «Aspirations, Inequality, Investment and Mobility». NBER Working Paper $\mathrm{N}^{\circ} 19976$. National Bureau of Economic Research.

KLIKSBERG, BERNARDO (2008): «Los desafíos éticos pendientes en un mundo paradojal. El rol de la universidad. Disertación acto de entrega del Doctorado Honoris Causa de la Universidad Rey Juan Carlos de España». Disponible en línea: www.redunirse.com.

KRAUSKOPF, DINA (2010): «La condición juvenil contemporánea en la constitución identitaria». Última Década N³3. Valparaíso: Ediciones CIDPA. (2011): «Ciudadanía y participación política de adolescentes jóvenes». Versión preliminar para publicar en Diagnóstico de los derechos de participación de las y los adolescentes en Chile. Santiago: UNICEF.

MidePlan, Costa RiCA (2010): Ministerio de Planificación Nacional y Política Económica de Costa Rica: Plan Nacional de Desarrollo 2011-2014. San José: Gobierno de Costa Rica. 
PRODUS (2011): «Megabase de datos georreferenciados para la educación primaria y secundaria de los centros educativos de Costa Rica (20002009)». Disponible en línea: www.estadonacion.or.cr.

PROGRAMA ESTADO DE LA NACIÓN (2011): III Estado de la educación. San José: Consejo Nacional de Rectores.

PROGRAMA ESTADO DE LA NACIÓN EN DESARROLLO HUMANO SOSTENIBLE (PEN) (2010): Decimosexto informe del estado de la nación. San José: El Programa.

PROGRAMA ESTADO DE LA NACIÓN EN DESARROLLO HUMANO SOSTENIBLE (PEN) (2008): Segundo estado de la educación. San José: Consejo Nacional de Rectores.

REgUILLO, RosSANA (1994): «Las tribus juveniles en tiempos de la modernidad». Estudios sobre las Culturas Contemporáneas, Vol. V, No015. México: Universidad de Colima.

SAINTOUT, Florencia (2007): «Jóvenes e incertidumbres. Percepciones de un tiempo de cambios: familia, escuela, trabajo y política». Tesis de Doctorado en Ciencias Sociales, FlACSO Argentina.

Sen, Amartya (2000): Desarrollo y libertad. Buenos Aires: Editorial Planeta.

SOJO, CARLOS (2006): «Pobreza, exclusión social y desarrollo. Visiones y aplicaciones en América Latina». Cuadernos de Ciencias Sociales Nº 142. San José: FLACSO Costa Rica. (2010): Igualiticos. La construcción social de la desigualdad en Costa Rica. San José: Master Litho.

UgaZ, CeCilia (2010a): «Clase 2: El enfoque del desarrollo humano y las capacidades». Maestría en Desarrollo Humano. Curso Desarrollo Humano, FLACSO Argentina.

VICUÑA, PILAR (2012): Muchachitas liceanas: La educación y la educanda del liceo fiscal femenino en Chile, 1890-1930. Santiago: Universidad de Chile. 